\title{
Homogeneity Bias in Models of Discrete Choice with Bounded Rationality
}

\author{
Russell Golman ${ }^{a *}$
}

August 25, 2011

${ }^{a}$ Department of Applied and Interdisciplinary Mathematics, University of Michigan, 2082 East Hall, 530 Church Street, Ann Arbor, MI 48109, USA. Telephone: 734 7647436. Fax: 734 763-0937. E-mail: rgolman@umich.edu

\footnotetext{
${ }^{*}$ I am grateful to Andreas Blass and Scott Page for many helpful conversations and to the anonymous referees for their valuable comments and suggestions. An earlier version of this paper was titled, "Logit Equilibria with Heterogeneous Agents." This work was funded by an NSF-IGERT grant.
} 


\begin{abstract}
Quantal response equilibrium captures bounded rationality in a strategic game by adopting a stochastic model of discrete choice along with the traditional rational expectations framework. We examine the use of a single-agent, homogeneous parametric quantal response model (e.g., logit response) to describe the aggregate behavior of heterogeneous agents sharing the same parametric form for their quantal response functions, but having individual rationality parameters, in a symmetric population game. For any parametric quantal response function arising from a unimodal distribution of exchangeable payoff disturbances, we find that a mis-specified homogeneous rationality parameter will have downward bias. Logit response is one such specification. This result implies that empirical work that disregards heterogeneity underestimates subjects' rationality.
\end{abstract}

KEYWORDS: logit equilibrium, quantal response equilibrium, bounded rationality, heterogeneity, logit response

JEL classification codes: C19, C44, C72 


\section{Introduction}

Discrete choice models have traditionally been interpreted as capturing heterogeneous preferences in an observationally homogeneous population (McFadden 1974). But stochastic models of choice, such as the logit model, can also be interpreted as capturing bounded rationality due to perceptual or implementational errors (Luce 1959; Anderson et al. 1992). We take the position that both heterogeneity and bounded rationality should be accounted for in models of choice. We assume that individual agents are boundedly rational and their behavior can thus be modeled with stochastic choice functions. Moreover, a population of such agents should be heterogeneous and thus contain agents who vary in their likelihoods of making bad choices.

We wish to consider as broad a category of discrete choice as possible, and so we allow situations in which the payoffs to agents in the population depend on everybody's choices. This puts us in the realm of normal-form population games. We can recover the single-player discrete choice environment in our analysis as a special case in which payoffs do not depend on the population state and the single player is a randomly selected agent. The game theoretic analogue of the logit model of discrete choice is the logit equilibrium (McKelvey and Palfrey 1995).

The logit equilibrium model has been shown to fit experimental data better than the Nash Equilibrium model of perfectly rational play in games (McKelvey and Palfrey 1995; Capra et al. 1999; Goeree and Holt 1999; Goeree et al. 2002; Fey et al. 1996; Anderson et al. 2002). Logit equilibrium extends the concept of Nash Equilib- 
rium by replacing sharp best reply functions with smooth logistic probabilistic choice functions. These logit response functions give players a positive probability of selecting any action. Logit responders can thus be seen as boundedly rational players, making errors while trying to choose optimal strategies. But, the likelihood of an error is inversely related to its cost. Logit response functions have one free parameter, interpretable as the player's degree of rationality. The higher this rationality parameter, the more likely the player is to select a best response.

Logit equilibrium is the most prominent parametric form of quantal response equilibrium, a state of play occurring at the intersection of stochastic best-response functions, or quantal response functions (McKelvey and Palfrey 1995; Goeree et al. 2005). Structural quantal response functions arise from random utility models in which players observe payoffs disturbed by idiosyncratic noise. Logit response, in particular, arises when payoff disturbances are all independently drawn from an extreme value distribution. ${ }^{1}$ This logit specification of choice satisfies independence of irrelevant alternatives and invariance under constant shifts of utility (Luce 1959).

In most experimental treatments a single logit parameter is estimated from the play of a pool of subjects. But, in a large population of agents, we should expect heterogeneity of behavior (Hommes 2006; Kirman 2006). A population of logit responders (or, more generally, any form of quantal responders) should consist of agents

\footnotetext{
${ }^{1}$ Logit response can also arise in models of players who have to make some effort in order to implement any desired strategy, but who perfectly maximize utility subject to this control cost (Mattsson and Weibull 2002).
} 
who may have different error rates. In fact, McKelvey et al. (2000) find experimental evidence for heterogeneous logit parameters in trying to fit data from two-by-two asymmetric games. ${ }^{2}$

Prior research in other directions has considered a distribution of logit responders with varying rationality parameters (Tsakas 2005; Rogers et al. 2009). Tsakas (2005) shows that heterogeneous mixed logit equilibrium models are sufficiently flexible to approximate other quantal response equilibrium models, and Rogers et al. (2009) finds that a heterogeneous logit model does indeed improve fit with data pooled across games. Our interest here is how a mis-specified homogeneous logit model misrepresents heterogeneous agents. A population of heterogeneous logit responders can be captured by a single representative agent, but that representative agent is not itself a logit responder (Golman 2011). Because the homogeneous logit model is mis-specified if agents are heterogeneous, it cannot explain equilibrium choice probabilities and payoffs in general. For a symmetric game with just two pure strategies, we can obtain analytically a homogeneous logit parameter to match the behavior of heterogeneous agents. In more general contexts, we can determine logit parameter values with maximum likelihood estimation. However, we should not expect consistency of our estimates of a mis-specified parameter across games. In order to make predictions across games, we should estimate a reduced-form representative-agent

\footnotetext{
${ }^{2}$ Further motivation to consider heterogeneous logit responders comes from recent findings that heterogeneity introduces bias in fitting the parameters of the experience-weighted attraction learning model to data (Wilcox 2006; Ho et al. 2008).
} 
model from data spanning the full range of payoffs (Golman 2011). Whereas Tsakas (2005), Rogers et al. (2009), and Golman (2011) show how heterogeneity should be accounted for in a quantal response equilibrium model, this paper analyzes the bias that emerges when heterogeneity is ignored. ${ }^{3}$

We consider a population of agents who occasionally err in their choice of strategy according to some (common) parametric form of quantal response (quite possibly logit, but perhaps probit or a reduced form instead), and we suppose this population is heterogeneous in that the agents vary in their rationality parameters. We compare the single-agent, homogeneous parametric quantal response model to the aggregate behavior of these heterogeneous quantal responders in the context of a fixed symmetric game with two pure strategies. In the case that the quantal response function satisfies a convexity condition corresponding to a unimodal distribution of payoff disturbances, we find that a mis-specified homogeneous rationality parameter will exhibit a downward bias. It will be less than the average (i.e., the arithmetic mean) of the true heterogeneous rationality parameters. This implies that empirical estimates mis-specify representative agents as less rational than the populations those agents represent. The paper also includes numerical results that support the analysis in contexts with higher-dimensional strategy spaces.

Nash proposed a population game interpretation of equilibrium in his unpublished

\footnotetext{
${ }^{3}$ In this sense, our analysis runs parallel to that of Wilcox (2006), which also describes a bias when heterogeneity is ignored, but in the context of the experience-weighted attraction learning model rather than the quantal response equilibrium model of bounded rationality in games.
} 
PhD dissertation (Weibull 1994). Following his lead, we assume that there is a population of agents who are randomly matched to play a symmetric game. We assume the population is large, and we are interested in the chance that a given strategy is played by a randomly selected member of the population. Each player in the original game is associated with an entire population of agents in the population game, so a mixed strategy relying on a randomizing device in the original game can be reinterpreted as the expected strategy of a population. We refer to a person in a population game as an agent, as distinguished from the player in the original game who is now associated with an entire population. The assumption of a single population for a symmetric game imposes the restriction that all players in the original game should play identical population mixed strategies because their roles are indistinguishable. For simplicity, we allow the possibility that an agent is matched to play against himself so that all agents face the same strategic context; this has a negligible effect in a large population. An agent's payoff is his expected payoff given the random matching of agents to play against.

Population games provide a framework for the use of evolutionary learning dynamics. In some important classes of games, a learning rule that assumes that players noisily best respond will converge to a logit equilibrium (Blume 1993; Fudenberg and Levine 1998; Hofbauer and Sandholm 2002; Anderson et al. 2004; Hofbauer and Hopkins 2005; Alos-Ferrer and Netzer 2010). This paper focuses on the equilibrium itself and not on the learning rules that might lead to it. Population games also describe experimental settings well, as data is accumulated through the randomly matched 
interactions of many subjects.

\section{Parametric Quantal Response Equilibrium}

Consider a symmetric normal form game with players randomly selected from a population of agents. (We can capture a discrete choice environment with additive random utility as a one-player game.) Let $S=\left\{s_{1}, \ldots, s_{J}\right\}$ be the set of pure strategies available to the agents. The collective play of all the agents in the population defines the population mixed strategy vector $x$. Formally, $x=\left(x_{1}, \ldots, x_{J}\right) \in \triangle^{J-1}$, the $(J-1)$-dimensional simplex where $x_{j} \geq 0$ for all $j$ and $\sum_{j} x_{j}=1$. We interpret $x_{j}$ as the expected proportion of plays of strategy $s_{j}$, or in other words, the chance that $s_{j}$ is used by a randomly selected member of the population. (As discussed below, in our model there are two sources for randomness in an opponent's strategy: random selection of an agent in the population and that agent's random selection of a pure strategy according to her individual mixed strategy. The population mixed strategy vector $x$ reflects both sources of randomness.)

In a population game, payoffs may depend on the expected frequency with which each strategy is played, but it does not matter which agents in a population take which strategies because by assumption, agents care only about their expected payoffs given random matching. All agents in a symmetric population game have the same payoff function. The vector $\pi=\pi_{1}, \ldots, \pi_{J}$ denotes the payoffs from choosing each of the pure strategies. Of course, we should write $\pi=\pi(x)$ to reflect the fact that payoffs 
are a function of the population mixed strategy vector describing what to expect from the opposing agents, but at times we omit the payoff function's argument for ease of notation. Formally, $\pi: \triangle^{J-1} \rightarrow \Re^{J}$.

In a traditional population game, the agents select pure strategies; heterogeneity of the agents is sufficient to generate mixed strategies at the population level. Here, however, we wish to describe a heterogeneous population of boundedly rational agents. Each agent should have some probability of making a mistake. So each agent has a quantal response function determining a mixed strategy. ${ }^{4}$

The response function for each agent returns the agent's likelihood of choosing each strategy given the agent's payoffs. Let $Q_{j}^{\mu}(\pi)$ be the probability that agent $\mu$ selects strategy $s_{j}$ given the payoffs to each strategy in $S$. We write $Q^{\mu}=\left(Q_{1}^{\mu}, \ldots, Q_{J}^{\mu}\right)$, and we have $Q^{\mu}: \Re^{J} \rightarrow \triangle^{J-1}$. For our analysis, the response function should satisfy regularity (i.e., choice probabilities are positive, continuous in the payoff vector, strictly increasing in own payoff, and monotonically ordered by payoff) and translation invariance (i.e., when a constant is added to all payoffs, the choice probabilities do not change), properties which follow from a structural assumption that the response function arises due to exchangeable disturbances (with full support and admissibil-

\footnotetext{
${ }^{4}$ Formally, a player obeying quantal response can be associated with a population of types, each with an unobservable component in its payoffs (see, e.g., Signorino 2003). Our approach to modeling a heterogeneous population of quantal responders is to use this trick twice over. Of course, the interpretation remains that when an agent obeys quantal response, she is making errors, not that agents are themselves composed of subpopulations.
} 
ity) added to payoffs (Goeree et al., 2005). We introduce a parameter $\lambda>0$ into the quantal response function so that it takes the form $Q(\pi)=\mathcal{Q}(\lambda \pi)$. The $\lambda$ can be interpreted as the agent's level of rationality. Higher values of $\lambda$ tend to produce choices that are closer to best responses. For the logit specification of quantal response in particular,

$$
\mathcal{Q}_{j}(\lambda \pi)=\frac{e^{\lambda \pi_{j}}}{\sum_{l=1}^{J} e^{\lambda \pi_{l}}} .
$$

The logit rationality parameter permits a clear illustration. As $\lambda$ goes to infinity, agents best respond perfectly. Conversely, as $\lambda$ tends to zero, agents play the uniform mixed strategy, choosing each action with the same probability without regard to payoffs.

The response functions for all the agents in the population can be aggregated to give the population mixed strategy response to any given population state. In a finite population of $m$ agents, the population aggregate response function is $\hat{Q}_{j}=$ $\frac{1}{m} \sum_{\mu=1}^{m} Q_{j}^{\mu}$ for all $j .^{5}$ An equilibrium is defined by the fixed point equation $x_{j}=$ $\hat{Q}_{j}(\pi(x))$ for all $j$. The equilibrium mixed strategy vector $x^{*}$ determines an equilibrium payoff vector $\pi^{*}=\pi\left(x^{*}\right)$ (and vice versa).

The conventional assumption that all agents have the same rationality parameter $\lambda$ yields a homogeneous parametric quantal response equilibrium.

Definition A homogeneous parametric quantal response equilibrium to a game with

\footnotetext{
${ }^{5}$ The reader concerned with the small but nonzero chance that an agent is matched to play against himself may take $m \rightarrow \infty$ here. While this is clearly an idealization, it poses no theoretical problems (Boylan, 1992; Gilboa and Matsui, 1992; Alos-Ferrer, 1999).
} 
payoff function $\pi$ is a mixed strategy vector $x^{*}$ that solves

$$
x=\mathcal{Q}(\lambda \pi(x)) .
$$

When the parametric quantal response function is logistic, as in Equation (1), we have the logit equilibrium.

Definition A (homogeneous) logit equilibrium with any given $\lambda \geq 0$ is a mixed strategy vector $x^{*}$ that solves

$$
x_{j}=\frac{e^{\lambda \pi_{j}(x)}}{\sum_{l=1}^{J} e^{\lambda \pi_{l}(x)}}
$$

for all $j$.

Whereas a Nash Equilibrium is a state of play with everybody simultaneously playing a best response, a logit equilibrium is a state with everybody simultaneously playing according to a logit response function. In particular, as $\lambda$ tends toward infinity, the logit equilibrium approaches the Nash Equilibrium.

We now suppose that agents' quantal response functions take a particular parametric form - we have in mind the logit form, but probit and many other forms are acceptable as well - and we recognize heterogeneity by allowing the agents to have their own individual rationality parameters. That is, we assume

$$
Q^{\mu}(\pi)=\mathcal{Q}\left(\lambda_{\mu} \pi\right)
$$

For the logit specification we would have

$$
\mathcal{Q}_{j}\left(\lambda_{\mu} \pi\right)=\frac{e^{\lambda_{\mu} \pi_{j}}}{\sum_{l=1}^{J} e^{\lambda_{\mu} \pi_{l}}} .
$$


Definition A mixed parametric quantal response equilibrium is a mixed strategy vector $x^{*}$ that solves

$$
x=\frac{1}{m} \sum_{\mu=1}^{m} \mathcal{Q}\left(\lambda_{\mu} \pi(x)\right) .
$$

Definition When Equation (5) holds, the mixed parametric quantal response equilibrium is called a (heterogeneous) mixed logit equilibrium (Tsakas 2005).

In a mixed logit equilibrium, agents are aware of the mixed strategy profile they face, or more generally, have knowledge of the distribution of heterogeneous logit parameters. This mixed strategy profile is then a fixed point of the population aggregate response function. The mixed logit equilibrium is distinct from Rogers et al.'s (2009) heterogeneous quantal response equilibrium (HQRE) in that it applies to symmetric population games as opposed to traditional (possibly asymmetric) games. We can naturally embed a symmetric population game in the traditional framework and given this perspective view a mixed logit equilibrium as a special case of HQRE with a loss of information. In a population game knowledge of the distribution of heterogeneous logit parameters is sufficient for an agent to deduce the equilibrium payoffs and the mixed strategy profile she will face, because agents care only about their expected payoffs with random matching. (In the more general framework of HQRE, agents can deduce expected payoffs and the expected mixed strategy profile in equilibrium, but the ex post equilibrium payoffs and mixed strategy profile after the realization of agents' types may be different.)

Our assumption of a common parametric form for all agents is a restriction not 
without loss of generality. ${ }^{6}$ Imposing this restriction lets us focus on bias caused by disregarding heterogeneity apart from any bias due to mis-specification of the functional form of one's quantal response rule. We are thus giving the analyst the benefit of the doubt by assuming correct specification of a functional form and then characterizing the bias that results from forcing a homogeneous model on heterogeneous agents.

\section{Downward Bias}

In this section, we see what happens when an analyst tries to force a homogeneous parametric quantal response equilibrium (e.g., a logit equilibrium) model on a population that is actually heterogeneous. We assume that the population is in a mixed parametric quantal response equilibrium. Because the homogeneous model is misspecified, the value of the single rationality parameter will vary with the game being considered. But, for a given game, any particular choice probabilities between two actions that preserves payoff monotonicity (i.e., with choice probabilities increasing in the payoffs) can be explained by a single homogeneous parameter (as long as the parametric form is capable of approximating a pure strategy, which must be the case for structural quantal responses arising from random payoff disturbances). For this reason, we restrict attention to a symmetric game with two pure strategies.

\footnotetext{
${ }^{6}$ Golman (2011) characterizes a heterogeneous population of quantal responders nonparametrically.
} 
We identify a downward bias in the single logit parameter determined by the mis-specified homogeneous model as compared with the average of the true logit parameters in use. Thus, the population seems to behave less rationally if the modeler believes the agents are all alike when in fact they each have their own levels of rationality. This bias is exacerbated as the magnitude of the difference in payoffs between the two actions grows. We describe the result here in the context of the logit specification because this functional form is so commonly employed in practice, but our results will hold for any stochastic choice function arising from a unimodal distribution of payoff disturbances.

Let $J=2$. Fix equilibrium choice probabilities and payoffs in accordance with a mixed parametric quantal response equilibrium (6), and denote them $x^{*}$ and $\pi^{*}$ respectively. Assume a game in which the equilibrium payoffs to the two actions are not equal, $\pi_{1}^{*} \neq \pi_{2}^{*}$. By translation invariance of the quantal response function, a sufficient statistic for the equilibrium payoffs is their difference $\Delta \pi=\pi_{1}^{*}-\pi_{2}^{*}$. Now we can express the quantal response in terms of the payoff difference,

$$
\mathcal{Q}\left(\lambda_{\mu} \pi^{*}\right)=\left(q\left(\lambda_{\mu} \Delta \pi\right), 1-q\left(\lambda_{\mu} \Delta \pi\right)\right),
$$

where we have introduced $q(\varpi)=\mathcal{Q}_{1}(\varpi, 0)$ to simplify notation going forward. Denote by $\lambda$ the rationality parameter of the homogeneous model that captures the equilibrium choice probabilities and payoffs of this heterogeneous population.

Theorem 1 says that for any specification of the quantal response function $\mathcal{Q}$ satisfying a convexity property corresponding to a unimodal distribution of payoff 
disturbances, the homogeneous rationality parameter is less than the average of the heterogeneous parameters actually used by the agents. Moreover, the size of this bias in the homogeneous model depends on the equilibrium payoffs. As the difference in payoffs between the two actions grows large, the biased homogeneous rationality parameter tends to a limit no greater than the geometric mean of the agents' true rationality parameters (which is, of course, always less than the arithmetic mean) and perhaps as small as the minimum of the heterogeneous parameters. On the other hand, when the magnitude of the payoff difference goes to zero, the homogeneous rationality parameter approaches the average of the agents' true rationality parameters. Thus, the bias disappears in this limit.

Theorem 1 Consider a mixed parametric quantal response equilibrium in accordance with Equation (6) such that the two pure strategies have different equilibrium payoffs, i.e., $J=2$, and $\Delta \pi \neq 0$. Let $\bar{\lambda}=\frac{1}{m} \sum_{\mu=1}^{m} \lambda_{\mu}$ be the average of the heterogeneous rationality parameters used by the agents, and let $\lambda$ be the homogeneous parameter that explains this heterogeneous population's choice probabilities and payoffs. If $q$ is convex on $(-\infty, 0)$ and concave on $(0, \infty)$, then

$$
\lambda \leq \bar{\lambda}
$$

with equality if and only if $\lambda_{1}=\lambda_{2}=\ldots=\lambda_{m}$. Additionally,

$$
\lim _{\Delta \pi \rightarrow \pm \infty} \lambda \in\left[\min \left\{\lambda_{\mu}\right\}, \prod_{\mu=1}^{m} \lambda_{\mu}{ }^{\frac{1}{m}}\right]
$$


and if the derivative $q^{\prime}(0)$ is finite, then

$$
\lim _{\Delta \pi \rightarrow 0} \lambda=\bar{\lambda} .
$$

Proof We can use Equations (2), (4), and (7) to express $\lambda$ in terms of the true heterogeneous rationality parameters and the equilibrium payoffs:

$$
\lambda=\frac{1}{\Delta \pi} q^{-1}\left(\frac{1}{m} \sum_{\mu=1}^{m} q\left(\lambda_{\mu} \Delta \pi\right)\right) .
$$

By the responsiveness property of quantal response functions, we know that the function $q$ has an inverse $q^{-1}$, which is also monotonically increasing.

Without loss of generality, suppose action 1 has the higher equilibrium payoff so that $\Delta \pi>0$. We apply Jensen's Inequality to determine that

$$
\frac{1}{m} \sum_{\mu=1}^{m} q\left(\lambda_{\mu} \Delta \pi\right) \leq q(\bar{\lambda} \Delta \pi)
$$

with equality if and only if $\lambda_{1}=\lambda_{2}=\ldots=\lambda_{m}$. The inequality is preserved when we apply $q^{-1}$ to both sides because this function is monotonically increasing. So,

$$
q^{-1}\left(\frac{1}{m} \sum_{\mu=1}^{m} q\left(\lambda_{\mu} \Delta \pi\right)\right) \leq \bar{\lambda} \Delta \pi .
$$

Putting this inequality together with Equation (11) and cancelling the $\Delta \pi$ leads us to $(8)$.

We prove the limits in (9) and (10) in the Appendix.

Theorem 1 describes a downward bias in the determination of a homogeneous rationality parameter when agents are actually heterogeneous. The less rational agents 
seem to leave a larger mark on the aggregate population behavior. The disproportionate impact the less rational agents have on aggregate behavior stems from the fact that the marginal effect on the probability of a mistake with respect to a changing rationality parameter is of greater magnitude for these agents.

Corollary 1 points out that Theorem 1 holds for logit parameters because the logit response function $\mathcal{Q}_{j}$ in Equation (1) is indeed convex in $\pi_{j}^{*}-\pi_{-j}^{*}$ on $(-\infty, 0)$ and concave on $(0, \infty)$ (and has a finite derivative at $\Delta \pi=0)$.

Corollary 1 Consider a mixed logit equilibrium in accordance with Equations (5) and (6) in the same context as in Theorem 1. Now let $\lambda$ be the analyst's determination of a homogeneous logit parameter rationalizing the mixed logit equilibrium choice probabilities and payoffs. Then $\lambda \leq \bar{\lambda}$ with equality if and only if $\lambda_{1}=\lambda_{2}=\ldots=\lambda_{m}$. Additionally, $\lim _{\Delta \pi \rightarrow 0} \lambda=\bar{\lambda}$. (Equation (9) will be refined in Proposition 1.)

For the logit specification, we can more precisely characterize the maximal bias when the payoff difference gets large, complementing our finding that the bias vanishes as the payoff difference gets small. Proposition 1 establishes that as the magnitude of the payoff difference grows, the homogeneous logit parameter approaches the smallest of the heterogeneous logit parameters in the population. In this limit, the population behaves like its single most irrational agent.

Proposition 1 Retain the context of Corollary 1. We have

$$
\lim _{\Delta \pi \rightarrow \pm \infty} \lambda=\min \left\{\lambda_{\mu}\right\}
$$


The proof of Proposition 1 is in the Appendix.

Our formula for $\lambda$, Equation (11), also allows us to ask whether a determination of the homogeneous rationality parameter from data on a choice between two actions restricts the set of possible rationality parameters for members of the population. The next result says it very well may. A large value of the homogeneous rationality parameter imposes a minimum possible value on the set of heterogeneous parameters. Conversely, a small homogeneous parameter precludes any individual agent from having too large a value. For intermediate homogeneous parameter values, however, we cannot rule out any parameters for a single agent. Naturally, these bounds depend on the population size and are much less restrictive for a large population.

Proposition 2 Retain the context of Theorem 1. If $q(\lambda|\Delta \pi|)>1-\frac{1}{2 m}$, then

$$
\min \left\{\lambda_{\mu}\right\} \geq \frac{1}{|\Delta \pi|} q^{-1}(m q(\lambda|\Delta \pi|)-(m-1)) .
$$

If $q(\lambda|\Delta \pi|)<\frac{m+1}{2 m}$, then

$$
\max \left\{\lambda_{\mu}\right\} \leq \frac{1}{|\Delta \pi|} q^{-1}\left(m q(\lambda|\Delta \pi|)-\left(\frac{m-1}{2}\right)\right) .
$$

Proof See Appendix.

Homogeneous logit parameters are estimated in much of the experimental literature on two-by-two games, although often with data pooled across many populations and many games. Proposition 2 applies to a homogeneous logit parameter calculated 
for a single given symmetric game. If we believe that agents use logit responses, but are heterogeneous in their levels of rationality, this proposition translates a misspecified homogeneous logit parameter into restrictions on the set of possible logit parameters in a finite population.

To illustrate these results, we can compare a homogeneous logit model fit to data in a two-by-two symmetric game to compatible heterogeneous logit models featuring two types of responders - one with a high rationality parameter and the other with a low one. To make the example as simple as possible, we assume exactly half the agents are of each type (though with the data coming from an experiment on 214 subjects, we have no reason to actually believe there are just two types). We consider Guyer and Rapoport's (1972) "No Conflict" game, Game \#6 in their series of experiments. The payoff matrix is:

\begin{tabular}{l}
\multicolumn{3}{c}{ No Conflict } \\
\\
$A_{2} \quad B_{2}$ \\
$A_{1} \quad 4,4 \quad 2,3$ \\
$B_{1} \quad 3,2 \quad 1,1$
\end{tabular}

The players have a dominant strategy choosing action $A$. Guyer and Rapoport observe action $A$ played $90 \%$ of the time. Choosing to model this as a homogeneous logit equilibrium, we have an equilibrium payoff difference $\Delta \pi=1$ (as the payoff to $A$ happens to always exceed the payoff to $B$ by one), and thus $\lambda=\ln (9)$ in accordance with Equation $3 .^{7}$

\footnotetext{
${ }^{7}$ Goeree and Holt (2004) estimate a homogeneous logit parameter from data pooled across 37
} 
Plugging $\Delta \pi=1$ and $\lambda=\ln (9)$ into Equation 11 (and specifying the logit functional form) produces an equation implicitly relating $\lambda_{\text {LOW }}$ and $\lambda_{\text {HIGH }}$. Figure 1 shows possible values of these heterogeneous logit parameters. Pairs of $\lambda_{\mathrm{LOW}}$ and $\lambda_{\mathrm{HIGH}}$ values are determined by fixed $x$-values in the graph. Larger $x$-values correspond to greater dispersion in the heterogeneous logit parameter values, but the scaling along this axis is arbitrary. We can see that the average of $\lambda_{\text {LOW }}$ and $\lambda_{\text {HIGH }}$ always exceeds $\ln (9)$, and the lower value is bounded below by $\ln (4)$ while the higher value may be arbitrarily large. Guyer and Rapoport's data thus puts a bound on how irrational the low-type agents can be, and they only approach this bound if the other agents are hyper-rational.

Because a homogeneous model is mis-specified in the presence of heterogeneity, estimates of a single rationality parameter do not translate across different game environments. For any given $\mathcal{Q}$, the analyst's parameter $\lambda$ may depend both on the true heterogeneous parameters $\left\{\lambda_{\mu}\right\}$ and the equilibrium payoff difference $\Delta \pi$, so we may write $\lambda\left(\left\{\lambda_{\mu}\right\}, \Delta \pi\right)$. Theorem 1 and Proposition 2 imply the following result, which tells us that an estimate of a homogeneous rationality parameter in a particular game environment places no restriction on such an estimate in an alternative game environment, even with a working assumption that agents' rationality levels are fixed across games. Theorem 2 states that the set of heterogeneous rationality parameters that is consistent with a given homogeneous parametric quantal response equilibrium games, including this one, from Guyer and Rapoport's study. We obtain a different value of the homogeneous logit parameter because we use data from just this one game. 


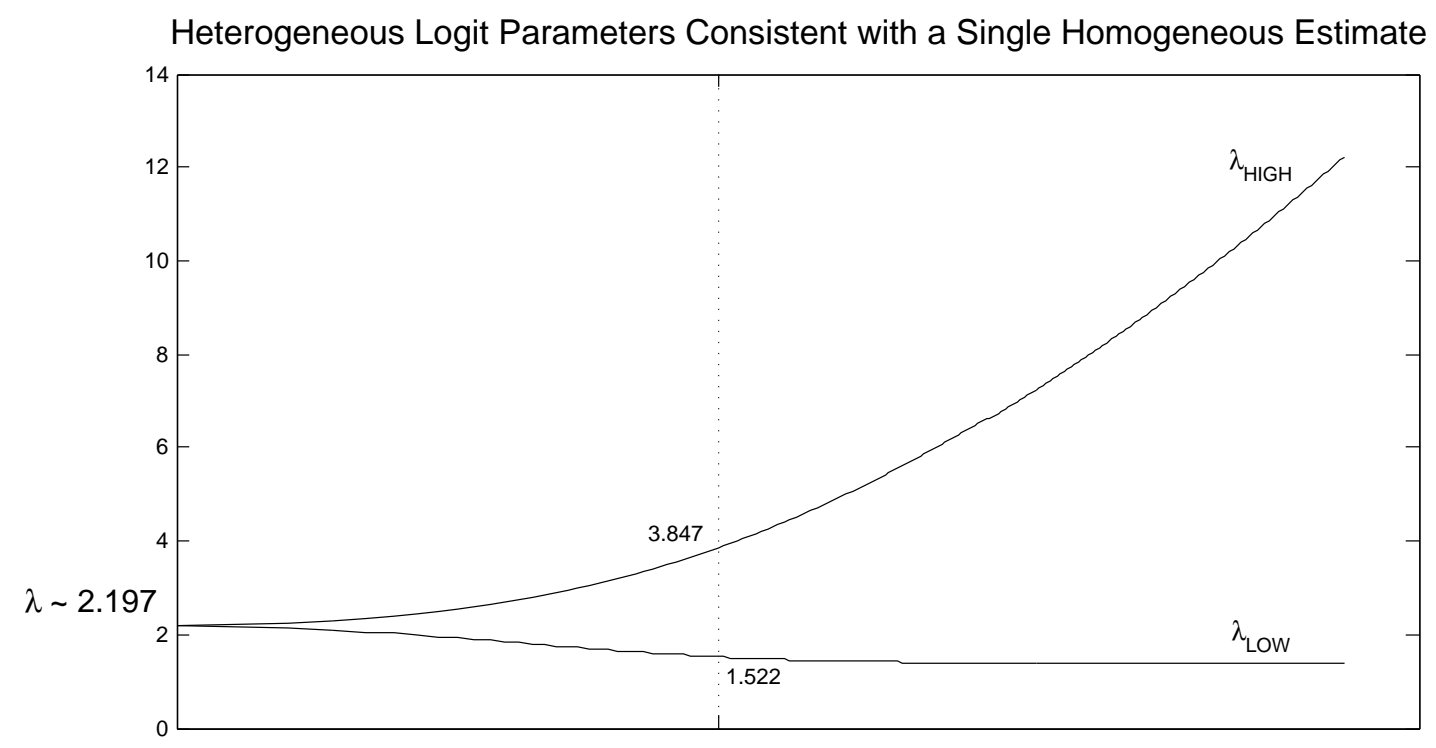

Figure 1: Possible values of a pair of logit parameters (determined at any fixed $x$ value) that would be consistent with a homogeneous $\lambda=\ln (9)$, when $\Delta \pi=1$. These values fit data from Guyer and Rapoport's (1972) "No Conflict" game.

in any one game could in some other game give rise to behavior consistent with any other homogeneous parameter.

Theorem 2 Consider symmetric normal form games with two pure strategies, $J=$ 2. Adopt any parametric specification with $q^{\prime}(0)$ finite and $q$ having full range (i.e., $\lim _{\varpi \rightarrow-\infty} q(\varpi)=0$ and $\lim _{\varpi \rightarrow \infty} q(\varpi)=1$ ). For any (mis-specified) homogeneous parametric quantal response equilibrium with payoff difference $\Delta \pi^{*} \neq 0$ and rationality parameter $\lambda^{*}>0$ in a game $\Gamma$, and any alternative value $\lambda^{\prime}>0$, there exists a set of heterogeneous rationality parameters $\left\{\lambda_{\mu}\right\}$ that are consistent with the homogeneous parametric quantal response model applied to $\Gamma$,

$$
\lambda\left(\left\{\lambda_{\mu}\right\}, \Delta \pi^{*}\right)=\lambda^{*},
$$

and there exists a game $\Gamma^{\prime}$ with a mixed parametric quantal response equilibrium with 
payoff difference $\Delta \pi^{\prime} \neq 0$ given heterogeneous parameters $\left\{\lambda_{\mu}\right\}$, such that

$$
\lambda\left(\left\{\lambda_{\mu}\right\}, \Delta \pi^{\prime}\right)=\lambda^{\prime} .
$$

Proof See Appendix.

Recall that Equation (11) for $\lambda\left(\left\{\lambda_{\mu}\right\}, \Delta \pi\right)$ gives us the homogeneous parameter that produces the same equilibrium choice probabilities as the heterogeneous rationality parameters $\left\{\lambda_{\mu}\right\}$ when the equilibrium payoff difference is $\Delta \pi$. Equation (13) means that any estimate of a homogeneous rationality parameter in a given game environment can be explained by some set of heterogeneous parameters, and Equation (14) means that these heterogeneous parameters could be consistent with any other homogeneous rationality parameter in an alternative game environment. We should not expect mis-specified parameter estimates to accurately describe behavior across all games.

We illustrate Theorem 2 with an example, again using Guyer and Rapaport's No Conflict game data as a jumping-off point and adopting the logit specification. As previously shown, an analyst fitting a homogeneous logit equilibrium to this data would find $\Delta \pi^{*}=1$ and $\lambda^{*}=\ln (9)$. What is the external validity of this finding? We do not know the true composition of the population, but we suspect it may be heterogeneous. Is it possible that we might analyze the same population playing a different game $\Gamma^{\prime}$ and find that a wildly different rationality parameter $\lambda^{\prime}$ is necessary 
for a homogeneous logit equilibrium to rationalize behavior in $\Gamma^{\prime}$ ? Theorem 2 answers this question in the affirmative. We might obtain any other $\lambda^{\prime}$. (We should be careful about stating our result precisely here. For any other $\lambda^{\prime}$, there is a heterogeneous composition of the population that we cannot rule out and there is a game $\Gamma^{\prime}$ in which the homogenous logit equilibrium with parameter $\lambda^{\prime}$ fits the data generated by this heterogeneous population. We are not asserting that we may obtain $\lambda^{\prime}$ regardless of the true composition of the population, nor are we asserting that in a particular game $\Gamma^{\prime}$, any $\lambda^{\prime}$ is possible.)

For our illustration, we show how $\lambda^{\prime} \approx 108$ might be obtained. We suppose $m$ is even and consider the possibility that $\lambda_{1}=\ldots=\lambda_{\frac{m}{2}} \equiv \lambda_{\text {LOW }}$ and $\lambda_{\frac{m}{2}+1}=\ldots=$ $\lambda_{m} \equiv \lambda_{\text {HIGH }}$. (To obtain a $\lambda^{\prime \prime} \approx 0$, we would have imposed a lower bound on $m$.) The values $\lambda_{\mathrm{HIGH}}=200 \ln (10) \approx 461$ and $\lambda_{\mathrm{LOW}}=\ln \left(4+\frac{25}{10^{200}-4}\right) \approx 1.39$ are consistent with $\Delta \pi^{*}=1$ and $\lambda^{*}=\ln (9)$ in the No Conflict game $\Gamma$, in accordance with the curves shown in Figure 1. If a population with this composition were to play the game $\Gamma^{\prime}$ with payoff function defined to be $\pi_{\Gamma^{\prime}}=\frac{1}{100} \pi_{\Gamma}$ (i.e., a version of the No Conflict game with payoffs scaled down by a factor of 0.01 ), then the homogeneous logit equilibrium would explain behavior in $\Gamma^{\prime}$ only if the rationality parameter were

$$
\lambda^{\prime}=100 \ln \left(\frac{201\left(4+\frac{25}{10^{200}-4}\right)^{.01}+100}{\left(4+\frac{25}{10^{200}-4}\right)^{.01}+102}\right) \approx 108
$$

as determined by Equation (11) under the logit specification.

So, do Guyer and Rapaport's data and an estimate of a homogeneous rationality parameter in a particular game $\Gamma$ offer us any predictive insight into behavior in an 
alternative game $\Gamma^{\prime}$ ? Yes. The responsiveness property of quantal response functions carries empirical content across games (Goeree et al. 2005; Haile et al. 2008). If we scale down the payoff function, we should see more errors. So, in the scaleddown No Conflict game $\Gamma^{\prime}$ above, we can predict that action $B$ (the mistake) will be played at least $10 \%$ of the time. But, we have not gained any additional information from estimating a mis-specified homogeneous rationality parameter. Without more information about the composition of the population, we do not know precisely how often action $B$ will be played in $\Gamma^{\prime}$. On the other hand, if we had fit a correctly specified representative-agent model of the heterogeneous population, the parameters would be consistent across all games (Golman 2011). A well-specified representativeagent model does make precise predictions across games.

\subsection{Maximum Likelihood Estimation}

We continue to focus on a single population in a fixed symmetric game, but we now allow more than two pure strategies. No longer can a homogeneous parametric quantal response model produce the same equilibrium choice probabilities and payoffs as the mixed parametric quantal response model. However, we can simulate equilibrium choice probabilities and payoffs for a set of heterogeneous rationality parameters and a given payoff matrix, and then determine a best fitting homogeneous parameter using maximum likelihood estimation. We proceed with the logit specification. In the vast majority of our simulations, the estimated homogeneous logit parameter is less than the average of the heterogeneous logit parameters. The effect is often quite large. 
For a symmetric game with $J \geq 2$, we take the payoff matrix $A$ as given, and for any set of heterogeneous logit parameters, we determine equilibrium choice probabilities $x^{*}$ and payoffs $\pi^{*}$ simultaneously using Equations (5) and (6) and $\pi^{*}=A\left(x^{*}\right)^{t r}$. The likelihood that a homogeneous logit model with rationality parameter $\lambda$ yields these choice probabilities in a sample of $T$ trials $(T \gg 1)$ is

$$
L(\lambda) \propto \prod_{j \in\{1 \cdots J\}}\left(x_{j}\right)^{x_{j}^{*} T}
$$

where the estimated choice probability $x_{j}$ is an implicit function of $\lambda$ defined by

$$
x_{j}=\frac{e^{\lambda \pi_{j}}}{\sum_{l=1}^{J} e^{\lambda \pi_{l}}}
$$

and $\pi=A x^{t r}$.

The first order condition for maximizing the log-likelihood is then

$$
\frac{d}{d \lambda} \ln (L(\lambda))=\sum_{j=1}^{J} T x_{j}^{*} x_{j}^{-1} \frac{d x_{j}}{d \lambda}=0 .
$$

We can solve for $\frac{d x}{d \lambda}$ by taking derivatives and using matrix algebra. Let $X$ be the diagonal matrix with $x_{1}, \ldots, x_{J}$ on the diagonal, $(X)_{i j}=x_{j} \delta_{i j}$, where $\delta_{i j}$ is the Kronecker delta. Also denote by $I$ the identity matrix, 1 a column vector of ones, and $\bar{\pi}$ the average payoff $x \cdot \pi$. Tedious algebra will give us

$$
\frac{d x}{d \lambda}=\left(I-\lambda X A+\lambda x^{t r} x A\right)^{-1} X(\pi-\mathbf{1} \bar{\pi}) .
$$

This can be plugged into Equation (16), making the first order condition for maximizing the log-likelihood

$$
\left(x^{*}\right) X^{-1}\left(I-\lambda X A+\lambda x^{t r} x A\right)^{-1} X(\pi-\mathbf{1} \bar{\pi})=0 .
$$


We solved Equation (17) (together with (15)) for $\lambda$ numerically using a variety of values for the heterogeneous logit parameters and the payoff matrix. In slightly over $93 \%$ of our trials, ${ }^{8}$ we found $\lambda$ to be less than the average of the heterogeneous logit parameters. On average across all trials, the estimated homogeneous logit parameter was $85 \%$ as large as the average of the heterogeneous parameters. Thus, it seems our finding of downward bias in the rationality parameter of the homogeneous model extends to choices between more than two pure strategies. ${ }^{9}$

\section{Discussion}

In the presence of heterogeneity, a homogeneous logit equilibrium model is misspecified. In the case of heterogeneous parametric quantal responders playing a symmetric game with two pure strategies, we have obtained a formula (Equation 11) relating a mis-specified homogeneous rationality parameter to the actual heteroge-

\footnotetext{
${ }^{8}$ We ran 8013 simulations and found $\lambda$ to be less than the average of the heterogeneous logit parameters in 7467 of the trials. The few exceptions arise because estimated choice probabilites and payoffs are determined simultaneously with the estimated homogeneous logit parameter, and deviations between the estimated behavior and the simulated equilibrium behavior can occasionally have a greater impact on $\lambda$ than the downward bias that results from ignoring heterogeneity. In additional trials with fixed payoffs, $\lambda$ was always less than the average of the heterogeneous logit parameters.

${ }^{9}$ We reviewed estimates made by Rogers et al. (2009) with a uniform distribution of types, and they too show the homogeneous logit parameter to always be less than the average of the heterogeneous parameters.
} 
neous parameters in the population. Maximum likelihood estimation can be used to fit a homogeneous parameter to the behavior of heterogeneous agents choosing between any number of pure strategies, but a closed form solution is not generally possible. Our formula provides insights in two directions. It tells us that the homogeneous model is biased towards less rationality, as a common convexity property guarantees that the homogeneous rationality parameter is less than the average of the heterogeneous ones. This bias gets worse when one action's equilibrium payoff gets much larger than the other's. Conversely, the bias disappears as the payoff difference tends to zero. The formula also allows us to bound the possible values of the true rationality parameters if we have a mis-specified homogeneous model already in place. This result is applicable to experimental work in which a homogeneous logit model has been fit to data. However, if enough distinct types are allowed, the bounds are very weak, possibly even non-existent. A mis-specified homogeneous model has no predictive power across games.

\section{Appendix}

\section{Completing the Proof of Theorem 1.}

We will show that $a$ ) when $\Delta \pi$ is large, $\lambda$ is monotonically decreasing in $\Delta \pi$, and

$b)$ in this limit, $\lambda$ is bounded within the range $\left[\min \left\{\lambda_{\mu}\right\}, \prod \lambda_{\mu}^{\frac{1}{m}}\right]$; and thus $\lambda$ must converge to some value in this range. The argument when $\Delta \pi$ is negative is completely analogous. 
We begin by asserting that since $q$ is monotonically increasing and bounded above by 1 , it must have a horizontal asymptote, and thus, for large enough $\varpi$, the composition $q\left(e^{\varpi}\right)$ is concave, and $\varpi q^{\prime}(\varpi)$ is decreasing and concave. Take a derivative of Equation (11) with respect to $\Delta \pi$ to obtain

$$
\frac{d \lambda}{d \Delta \pi}=\frac{1}{\Delta \pi}\left[\frac{1}{q^{\prime}(\lambda \Delta \pi)} \frac{1}{m} \sum_{\mu=1}^{m} \lambda_{\mu} q^{\prime}\left(\lambda_{\mu} \Delta \pi\right)-\lambda\right] .
$$

By Jensen's Inequality and the fact that $\varpi q^{\prime}(\varpi)$ is concave for $\varpi$ sufficiently large,

$$
\frac{1}{m} \sum_{\mu=1}^{m} \lambda_{\mu} q^{\prime}\left(\lambda_{\mu} \Delta \pi\right) \leq \bar{\lambda} q^{\prime}(\bar{\lambda} \Delta \pi)
$$

when $\Delta \pi$ is large enough. For large $\Delta \pi$ we also have

$$
\lambda q^{\prime}(\lambda \Delta \pi) \geq \bar{\lambda} q^{\prime}(\bar{\lambda} \Delta \pi)
$$

because $\lambda \leq \bar{\lambda}$ and $\varpi q^{\prime}(\varpi)$ is decreasing on this domain. Together, these two inequalities imply $\frac{d \lambda}{d \Delta \pi} \leq 0$ for sufficiently large $\Delta \pi$.

To establish the upper bound on $\lim _{\Delta \pi \rightarrow \infty} \lambda$, we use Jensen's inequality on $q\left(e^{\varpi}\right)$ with $\varpi$ taking values of $\ln \left(\lambda_{\mu} \Delta \pi\right)$. By the concavity of $q\left(e^{\varpi}\right)$ for such values, when $\Delta \pi$ is large,

$$
\begin{aligned}
\frac{1}{m} \sum_{\mu=1}^{m} q\left(\lambda_{\mu} \Delta \pi\right) & \leq q\left(e^{\frac{1}{m} \sum_{\mu=1}^{m} \ln \left(\lambda_{\mu} \Delta \pi\right)}\right) \\
& =q\left(\prod \lambda_{\mu^{\frac{1}{m}} \Delta \pi}\right) .
\end{aligned}
$$

Straightforward manipulation with Equation (11) produces the desired upper bound. The lower bound follows immediately from the monotonicity of $q$ and $q^{-1}$ in Equation (11) when we consider $\lambda_{\mu} \geq \lambda_{\min }$ for all $\mu$. 
To obtain the limit as $\Delta \pi$ approaches 0 , we apply l'Hospital's Rule to the expression for $\lambda$ given in Equation (11). We have

$$
\frac{d}{d \Delta \pi}\left[q^{-1}\left(\frac{1}{m} \sum_{\mu=1}^{m} q\left(\lambda_{\mu} \Delta \pi\right)\right)\right]=\frac{1}{q^{\prime}\left(q^{-1}\left(\frac{1}{m} \sum_{\mu=1}^{m} q\left(\lambda_{\mu} \Delta \pi\right)\right)\right)} \frac{1}{m} \sum_{\mu=1}^{m} q^{\prime}\left(\lambda_{\mu} \Delta \pi\right) \lambda_{\mu} .
$$

Evaluating this derivative at $\Delta \pi=0$ is possible when $q^{\prime}(0)$ is finite. (We know $q(0)=\frac{1}{2}$ by the monotonicity property of quantal response functions, and $q^{\prime}(0)>0$ by the responsiveness property.) In this case, we can cancel $q^{\prime}(0)$ from the numerator and the denominator to obtain

$$
\begin{aligned}
\frac{d}{d \Delta \pi}\left[q^{-1}\left(\frac{1}{m} \sum_{\mu=1}^{m} q\left(\lambda_{\mu} \Delta \pi\right)\right)\right]_{\Delta \pi=0} & =\frac{1}{m} \sum_{\mu=1}^{m} \lambda_{\mu} \\
& =\bar{\lambda} .
\end{aligned}
$$

The denominator in (11) is $\Delta \pi$, so its derivative is 1 . Thus,

$$
\lim _{\Delta \pi \rightarrow 0} \lambda=\bar{\lambda}
$$

\section{Proof of Proposition 1.}

To obtain

$$
\lim _{\Delta \pi \rightarrow \pm \infty} \lambda=\min \left\{\lambda_{\mu}\right\}
$$

we take the limit as $\Delta \pi$ goes to $\infty$. By symmetry, the result then holds when $\Delta \pi$ goes to $-\infty$ as well. First, we use algebra to derive from Equations (1) and (11) the following expression for $\lambda$ :

$$
\lambda=\frac{1}{\Delta \pi} \ln \left(\frac{\sum_{\mu=1}^{m} e^{\lambda_{\mu} \Delta \pi} \prod_{\omega \neq \mu}\left(e^{\lambda_{\omega} \Delta \pi}+1\right)}{\sum_{\mu=1}^{m} \prod_{\omega \neq \mu}\left(e^{\lambda_{\omega} \Delta \pi}+1\right)}\right) .
$$


In the limit of $\Delta \pi$ going to $\infty$,

$$
\begin{aligned}
e^{\lambda_{\mu} \Delta \pi} \prod_{\omega \neq \mu}\left(e^{\lambda_{\omega} \Delta \pi}+1\right) & \rightarrow e^{\lambda_{\mu} \Delta \pi} \prod_{\omega \neq \mu} e^{\lambda_{\omega} \Delta \pi} \\
& =\prod_{\omega} e^{\lambda_{\omega} \Delta \pi}
\end{aligned}
$$

and

$$
\sum_{\mu=1}^{m} \prod_{\omega \neq \mu}\left(e^{\lambda_{\omega} \Delta \pi}+1\right) \rightarrow \prod_{\omega \neq \arg \min \left\{\lambda_{\mu}\right\}} e^{\lambda_{\omega} \Delta \pi}
$$

Thus,

$$
\begin{aligned}
\lim _{\Delta \pi \rightarrow \infty} \lambda & =\lim _{\Delta \pi \rightarrow \infty} \frac{1}{\Delta \pi} \ln \left(\frac{\sum_{\mu=1}^{m} \prod_{\omega} e^{\lambda_{\omega} \Delta \pi}}{\prod_{\omega \neq \arg \min \left\{\lambda_{\mu}\right\}} e^{\lambda_{\omega} \Delta \pi}}\right) \\
& =\lim _{\Delta \pi \rightarrow \infty} \frac{1}{\Delta \pi} \ln \left(m e^{\min \left\{\lambda_{\mu}\right\} \Delta \pi}\right) \\
& =\lim _{\Delta \pi \rightarrow \infty} \frac{\min \left\{\lambda_{\mu}\right\} \Delta \pi+\ln (m)}{\Delta \pi} \\
& =\min \left\{\lambda_{\mu}\right\} .
\end{aligned}
$$

\section{Proof of Proposition 2.}

Without loss of generality, assume $\Delta \pi>0$. For the homogeneous model to match the mixed parametric quantal response equilibrium, we require

$$
q(\lambda \Delta \pi)=\frac{1}{m} \sum_{\mu=1}^{m} q\left(\lambda_{\mu} \Delta \pi\right)
$$

in accordance with Equation (11). To obtain the lower bound on $\min \left\{\lambda_{\mu}\right\}$, we recognize that $q\left(\lambda_{\mu} \Delta \pi\right) \leq 1$ for all $\mu \neq \arg \min \left\{\lambda_{\omega}\right\}$. Applying these inequalities with some straightforward algebra gives us the desired lower bound. Note that this bound is meaningful only if $m q(\lambda \Delta \pi)-(m-1)>\frac{1}{2}$. To obtain the upper bound on $\max \left\{\lambda_{\mu}\right\}$, 
we recognize that $q\left(\lambda_{\mu} \Delta \pi\right) \geq \frac{1}{2}$ for all $\mu \neq \arg \max \left\{\lambda_{\omega}\right\}$ (given that $\Delta \pi>0$ ). Once again, straightforward algebra leads to the desired bound, which in this case is meaningful only if $m q(\lambda \Delta \pi)-\left(\frac{m-1}{2}\right)<1$.

\section{Proof of Theorem 2.}

Without loss of generality, assume $\Delta \pi^{*}>0$. Choose $m \in \mathbb{N}$ such that $m>$ $\frac{1}{2\left(1-q\left(\lambda^{*} \Delta \pi^{*}\right)\right)}$ and $m>\frac{1}{2 q\left(\lambda^{*} \Delta \pi^{*}\right)-1}$. This ensures that neither of the bounds in Proposition 2 apply. Thus, we can choose $\lambda_{m} \equiv \max \left\{\lambda_{\mu}\right\}$ such that

$$
\lambda_{m}>\frac{1}{\Delta \pi^{*}} q^{-1}\left(\frac{m q\left(\lambda^{*} \Delta \pi^{*}\right)-\frac{1}{2}}{m-1}\right)
$$

and $\lambda_{m}>m \lambda^{\prime}$, and then we can choose $\lambda_{1}<\frac{\lambda^{\prime m}}{\lambda_{m}{ }^{m-1}}$ and the remaining $\left\{\lambda_{\mu}\right\}$, for $\mu=2 \ldots m-1$, such that Equation (13) holds. That means these heterogeneous rationality parameters will be consistent with the homogeneous parametric quantal response equilibrium with rationality parameter $\lambda^{*}$ and equilibrium payoff difference $\Delta \pi^{*}$. We have specifically chosen $\lambda_{m}$ and $\lambda_{1}$ so that $\prod \lambda_{\mu}^{\frac{1}{m}}<\lambda^{\prime}<\bar{\lambda}$. Thus, noting the limits we take in Theorem 1, we establish that $\lambda\left(\left\{\lambda_{\mu}\right\}, \Delta \pi\right)$ is above $\lambda^{\prime}$ when $\Delta \pi \approx 0$ and is below $\lambda^{\prime}$ when $\Delta \pi$ is large. Because $\lambda\left(\left\{\lambda_{\mu}\right\}, \Delta \pi\right)$ is continuous in $\Delta \pi$, according to the intermediate value theorem there is some $\Delta \pi^{\prime}$ for which $\lambda\left(\left\{\lambda_{\mu}\right\}, \Delta \pi^{\prime}\right)=\lambda^{\prime}$. 


\section{References}

[1] Alos-Ferrer, C. (1999) Dynamical Systems with a Continuum of Randomly Matched Agents. Journal of Economic Theory 86, 245-267.

[2] Alos-Ferrer, C., and Netzer, N. (2010) The Logit Response Dynamics. Games and Economic Behavior 68, 413-427.

[3] Anderson, S., De Palma, A., and Thisse, JF. (1992) Discrete Choice Theory of Product Differentiation. MIT Press, Cambridge, MA.

[4] Anderson, S., Goeree, J., and Holt, C. (2002) The Logit Equilibrium: A Perspective on Intuitive Behavioral Anomalies. Southern Economic Journal 69(1), $21-47$.

[5] Anderson, S., Goeree, J., and Holt, C. (2004) Noisy Directional Learning and the Logit Equilibrium. Scandinavian Journal of Economics 106(3), 581-602.

[6] Blume, L. (1993) The Statistical Mechanics of Strategic Interaction. Games and Economic Behavior 5, 387-424.

[7] Boylan, R. (1992) Laws of Large Numbers for Dynamical Systems with Randomly Matched Individuals. Journal of Economic Theory 57, 473-504.

[8] Capra, M., Goeree, J., Gomez, R., and Holt, C. (1999) Anomalous Behavior in a Traveler's Dilemma? American Economic Review 89(3), 678-690. 
[9] Fey, M., McKelvey, R., and Palfrey, T. (1996) An Experimental Study of Constant-Sum Centipede Games. International Journal of Game Theory 25(3), 269-287.

[10] Fudenberg, D., and Levine, D. (1998) The Theory of Learning in Games. MIT Press, Cambridge, MA.

[11] Gilboa, I., Matsui, A. (1992) A Model of Random Matching. Journal of Mathematical Economics 21, 185-197.

[12] Goeree, J., and Holt, C. (1999) Stochastic Game Theory: For Playing Games, Not Just for Doing Theory. Proceedings of the National Academy of Sciences 96(19), 10564-10567.

[13] Goeree, J., and Holt, C. (2004) A Model of Noisy Introspection. Games and Economic Behavior 46(2), 365-382.

[14] Goeree, J., Holt, C., and Palfrey, T. (2002) Quantal Response Equilibrium and Overbidding in Private-Value Auctions. Journal of Economic Theory 104(1), 247272.

[15] Goeree, J., Holt, C., and Palfrey, T. (2005) Regular Quantal Response Equilibrium. Experimental Economics 8, 347-367.

[16] Golman, R. (2011) Quantal Response Equilibria with Heterogeneous Agents. Journal of Economic Theory . 
[17] Guyer, M., and Rapoport, A. (1972) 2x2 Games Played Once. The Journal of Conflict Resolution 16(3), 409-431.

[18] Haile, P., Hortacsu, A., and Kosenok, G. (2008) On the Empirical Content of Quantal Response Equilibrium. American Economic Review 98(1), 180-200.

[19] Ho, T., Wang, X., and Camerer, C. (2008) Individual Differences in EWA Learning with Partial Payoff Information. The Economic Journal 118, 37-59.

[20] Hofbauer, J., and Hopkins, E. (2005) Learning in Perturbed Asymmetric Games. Games and Economic Behavior 52(1), 133-152.

[21] Hofbauer, J., and Sandholm, W.H. (2002) On the Global Convergence of Stochastic Fictitious Play. Econometrica 70, 2265-2294.

[22] Hommes, C. (2006) Heterogeneous Agent Models in Economics and Finance. In: Judd, K., Tesfatsion, L. (eds) Handbook of Computational Economics, vol 2, Agent-Based Computational Economics. North Holland, Amsterdam.

[23] Kirman, A. (2006) Heterogeneity in Economics. Journal of Economic Interaction and Coordination 1, 89-117.

[24] Luce, D. (1959) Individual Choice Behavior. Wiley, New York.

[25] Mattsson, L., and Weibull, J. (2002) Probabilistic Choice and Procedurally Bounded Rationality. Games and Economic Behavior 41, 61-78. 
[26] McFadden, D. (1974) Conditional Logit Analysis of Qualitative Choice Behavior. Frontiers of Econometrics. Academic Press, New York, NY.

[27] McKelvey, R., and Palfrey, T. (1995) Quantal Response Equilibria for Normal Form Games. Games and Economic Behavior 10(1), 6-38.

[28] McKelvey, R., Palfrey, T., and Weber, R. (2000) The effects of payoff magnitude and heterogeneity on behavior in $2 \mathrm{x} 2$ games with unique mixed strategy equilibria. Journal of Economic Behavior \&3 Organization 42, 523-548.

[29] Rogers, B., Palfrey, T., and Camerer, C. (2009) Heterogeneous Quantal Response Equilibrium and Cognitive Hierarchies. Journal of Economic Theory 144 (4), 1440-1467.

[30] Signorino, C. (2003) Structure and Uncertainty in Discrete Choice Models. Political Analysis 11, 316-344.

[31] Tsakas, E. (2005) Mixed Quantal Response Equilibria for Normal Form Games. Paper presented at the International Conference on Game Theory.

[32] Weibull, J. (1994) The Mass-Action interpretation of Nash equilibrium. Paper presented at Nobel symposium in honor of John Nash.

[33] Wilcox, N. (2006) Theories of Learning in Games and Heterogeneity Bias. Econometrica 74, 1271-1292. 